\title{
DYNAMIC ANALYSIS OF THE WAITING AREA IN A PUBLIC STATION
}

\author{
Javier Otamendi \\ Universidad Rey Juan Carlos, Campus Vicálvaro \\ Facultad de Ciencias Jurídicas y Sociales \\ Departamento Economía Aplicada I \\ Paseo Astilleros s/n \\ 28032 Madrid, Spain \\ E-mail: franciscojavier.otamendi@urjc.es
}

\author{
José Manuel Pastor \\ Universidad Castilla La Mancha \\ Escuela Universitaria Politécnica de Cuenca \\ Departamento de Sistemas Informáticos \\ Campus Universitario s/n \\ 16071 Cuenca, Spain \\ E-mail: josemanuel.pastor@uclm.es
}

\section{KEYWORDS \\ Dynamics of Flow, Queueing, Simulation.}

\begin{abstract}
An application of simulation in the design of public transportation facilities is presented. The flow of passengers is modeled using discrete-event simulation, which facilitates the abstraction of the emptying and filling of the passengers into the buses, rather than the more usual continuous simulation for crowd movement. The decision variables include not only the physical layout of the queues but also some tactical variables which will help in future negotiations with labor unions and owners of the lines. A sensitivity analysis is performed to understand the influence of peaks of demands.
\end{abstract}

\section{INTRODUCTION}

Among the design activities of one of the future underground bus stations in Madrid, it is necessary to study the capacity of the waiting area so that the formation of waiting queues does not interfere with the usual movement of the users of the facilities. The design of the building and the individual platforms is being performed from a static, architectural point of view, so there is also the necessity to validate the design from a dynamic point of view.

Within the Technical Specifications Document, there is the necessity to develop mathematical models to perform the validation stage, specifically to estimate the maximum length of the waiting queues at the gates. The input values come from historic data about demand, frequency, capacity of the buses and some decisions about the management of the line.

The article starts with a description of the real system as designed by the architects. Secondly, the objectives are specified. Then, the data that has been collected from the public administration is included. The final model is then described, which includes the necessary validation step. There is a separate section for the decision variables that affect the design. The last two sections include the results of the simulation runs, both of the actual situation and of the sensitivity analysis performed.

\section{THE SYSTEM}

Let us start with a brief description of the facilities (Figure 1) and the flow of its users.

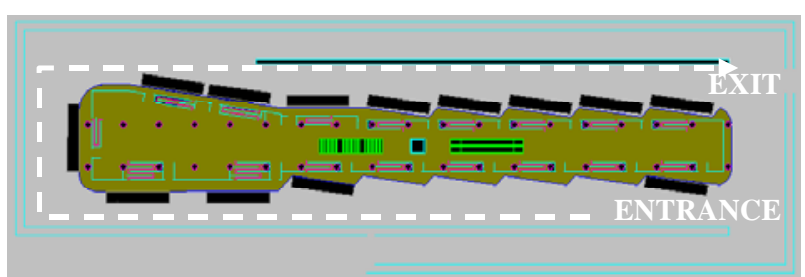

Figure 1. Layout

The bus station consists of a platform with 16 gates (depicted with a rectangle) with the possibility of parking 12-meter or 15-meter buses. The direction of movement of the vehicles is clockwise with the entrance and exit in the right hand side of the premises.

In front of each of the gates, queues of users are formed in zigzag (Figure 2). The layout of the queues has this shape for three main reasons. First, the movement within the platform is facilitated since the queue should never reach the center of the platform, thus blocking the movement. Second, there is a psychological factor that is improved since the rate of movement of the queue looks quicker than the actual rate to the people in it since users are moving in opposite directions. Third, this disposition also helps the flow of passengers moving in and out of the buses, since room is specifically dedicated to the output flows.

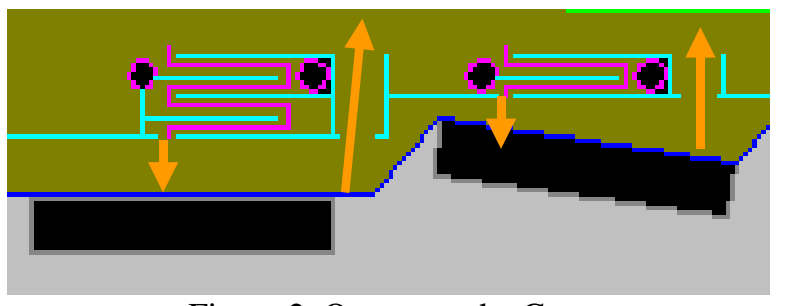

Figure 2. Queues at the Gates

To access the platform, the passengers have to use either a mechanical stair or a normal one, which are located in the center of the platform to reduce the 
distance between the stairs and the gates. The same set of stairs is used to leave the premises.

\section{OBJECTIVES}

The main objective is to measure the length of the queues. The desire is that it does not grow long enough to affect the flow in the center of the platform. That is, once a passenger gets to the bottom of the stairways, it should have a free path towards its departing gate.

The maximum number of waiting passengers is set to 56 , which is the capacity of a zigzag queue with four rows of 14 passengers each. This number is also similar to the capacity of the small-size buses that are often used. This queue size also agrees with the area in $\mathrm{m}^{2}$ of the designed platform.

This objective is going to be measured by monitoring the queue length every thirty seconds and plotting the values in a timeseries. There will be a separate graph for each of the gates.

The other objective could be the time the passengers spend in the queue. However, this measure depends on the interarrival time of the buses, which is not a design variable but input data. If a passenger is able to get into the next arriving bus, there will be no complaints.

\section{INPUT DATA}

\section{Bus Capacity}

The 12-meter buses have a capacity of 55 and the 15meter ones might hold up to 71 passengers, if they all are seated. Some 15-meter buses, the so-called mixed buses, might contain 134 users (46 seated and 88 standing).

\section{Demand}

The prediction for the next two decades is that thirteen bus lines will have a stop at this underground station, even if there is room for 16 gates.

Table 1 shows the data included in the Technical Specifications Document. Each row or record corresponds with one of the thirteen bus lines. The explanation of the columns is:

- PHI: PeakHourInterval. It is the time in minutes between consecutive departs

- PeakHour: hour of the day at which the peak hour occurs

- Buses: number of departs in the peak hour

- DailyDemand (arrivals + departures): total volume of users of the facilities

- DailyDepartures

- DailyArrivals

- HourlyRate: daily data obtained from on-site sampling:
- Arrivals: peak rate
- Departures: peak rate
- Capacity: maximum cumulative of arrivals and departures.

From these hourly and daily dates, the variable PeakPercentage is calculated. This variable is the percentage of DailyDepartures at the PeakHour, and it is used to calculate the HourlyRates for those lines for which only daily rates are available. As this percentage varies between $6.72 \%$ for line 4 and $11.4 \%$ for line 9 , this limit is taken for safe calculations (it is also the maximum likelihood estimator).

Table 1. Input Data

\begin{tabular}{|c|c|c|c|c|c|c|c|c|c|c|}
\hline & $\begin{array}{l}\text { Peak Hour } \\
\text { Interval }\end{array}$ & Peak Hour & Buses & Daily Demand & Daily Departures & Daily Arrivals & & & & \\
\hline & & & & & & & & Hourly Demand & & Peak Percentage \\
\hline & PHI & Departs & & & DD & DA & Arrivals & Departures & Capacity & \\
\hline 1 & 12 & 10 & 5 & 3068 & 2609 & 968 & & & & \\
\hline 2 & 6 & 8 & 10 & 6186 & 2284 & 1827 & & & & \\
\hline 3 & 20 & 7 & 3 & 1365 & 1248 & 1075 & & 131 & 160 & 0.1050 \\
\hline 4 & 20 & 14 & 3 & 1849 & 1324 & & 77 & 89 & 113 & 0.0672 \\
\hline 5 & 15 & 14 & 4 & 2843 & 1501 & 1269 & 149 & 162 & 212 & 0.1079 \\
\hline 6 & 30 & 15 & 2 & 1035 & 727 & & 102 & 71 & 115 & 0.0977 \\
\hline 7 & 30 & & 2 & 736 & 441.6 & & & & & \\
\hline 8 & 30 & & 2 & 934 & 560.4 & & & & & \\
\hline 9 & 12 & 15 & 5 & 6295 & 2009 & 1638 & 245 & 229 & 275 & 0.1140 \\
\hline 10 & 20 & & 3 & 1888 & 260 & 497 & & & & \\
\hline 11 & 12 & 14 & 5 & 7947 & 2199 & 1908 & 264 & 223 & 300 & 0.1014 \\
\hline 12 & 30 & & 2 & 3519 & 2111.4 & & & & & \\
\hline 13 & 15 & & 4 & 3529 & 2117.4 & & & & & \\
\hline
\end{tabular}




\section{Operation Times}

This section contains the rest of the necessary data to include in the simulation model. The corresponding values have been estimated:

- GateOpening: amount of time prior to departure in which the gates open for the users to jump into the bus. It is calculated as the time that is required to fill the whole bus.

- $\quad$ Bus Speed = 0.004 minutes/meter $(15 \mathrm{~km} / \mathrm{hr})$

- PassengerVelocity $=0.020$ minutes $/$ meter $(3$ $\mathrm{km} / \mathrm{hr}$ )

- $\quad$ FillRate $=20 \mathrm{pax} / \mathrm{min}$

- $\quad$ EmptyRate $=50 \mathrm{pax} / \mathrm{min}$

\section{THE SIMULATION MODEL}

Design of facilities considering pedestrian flow is one of the areas in which simulation fits perfectly ( $\mathrm{Li} 2000$, Hanish et al 2003, Hoogendorm and Daamaen 2004) as the mathematical model to use, not only due to its very good representation capabilities but also due to its exceptional experimentation possibilities in a design phase. In this particular case, although the model should have been used more in the earlier stages of the design, it is mainly used to validate the model from a dynamic point of view.

Even if continuous modeling looks to be the appropriate choice to model a dynamic system involving the movement of crowds (Chalmet et al 1982, Bruzzone and Signorile 1999, Teknomo 2005, Shen 2005), it is sometimes possible to use a discreteevent approach (Otamendi 2005). If the logic gets too complex, the continuous models have to include events to model the discontinuities. If the modeler feels more comfortable with the discrete-event approach, it should also be tried, especially with problems of small size.

\section{Description of the Model}

The buses are generated according to the peak hour intervals, directing them towards a preassigned gate, where the processes of emptying and filling take place.

The emptying operation takes place as soon as the bus gets to the gate and the doors open. The number of passengers in the arriving bus is calculated as follows:

$$
\text { ArrivingPax }=\text { DailyArrivals } * \frac{\text { PeakPercentage }}{\text { Buses }}
$$

When all the passengers have gotten out of the bus, the doors are closed. Several minutes later, at a predefined time prior to departure, Gate Opening, the doors open again and the passengers start to fill the bus. The rate of passengers is assumed constant and it is calculated as follows:

$$
\begin{aligned}
& \text { DepartingPax }=\text { ArrivingPax } \\
& \text { DepartingRate }=\frac{60}{\text { DepartingPax }}
\end{aligned}
$$

The bus then departs at its corresponding time with no delays.

\section{Hypothesis}

To facilitate this modeling step, the following restrictions are imposed:

- The peak hour coincides for any bus line.

- The simulation period is 5 hours (300 minutes), with the peak hour being the middle hour, the two contiguous hours with a rate of $50 \%$ of the peak rate and the other two with a rate of $25 \%$ of the peak (Figure 3 ).

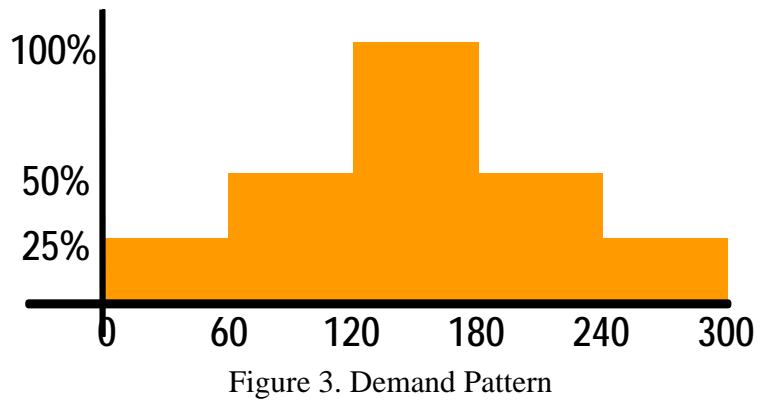

- The demand does not fluctuate within each hour.

- The arriving passengers leave the premises, that is, they do not take another bus.

- The departing passengers access directly to the queue, without waiting in the lounges.

- There are no delays with respect to the arriving or departing schedule.

The manager of the facilities has accepted these hypotheses since the behavior of the system is still well represented. The model is therefore validated and ready for experimentation.

\section{DECISION VARIABLES}

The parameters that a priori might affect the length of the queues, are:

- PeakPercentage: with an initial estimated value of $11.5 \%$.

- BusType: the three possibilities have already been mentioned: 12-meters, 15-meters (all seated) or mixed (15-meters with some standing passengers).

- PeakInterval: with the initial values set by contract and included in the specifications.

- GateOpening: with initial values corresponding to the necessary time to fill the bus with passengers. 
None of this variables are directly controlled at this validation stage by the management of the station. The PeakPercentage has been estimated from current data and are clearly influenced by uncontrolled factors, like weather, holidays... Maximum quantities, like peak values, are usually underestimated. To correct the bias, and foresee possible congestions, the management wants to know the behavior of the system for an increase of the peak percentage to $15 \%$.

The BusType is controlled by the owner of the line, which rents the facilities. In that sense, the management wants to know if they should force the owners to use different types of buses. The PeakInterval has been negotiated with the owners of the lines. A new negotiation might be initiated.

The opening of the gate is controlled by the drivers, with the time being agreed with the labor unions. The management wants to know however how they can negotiate with the drivers in order to improve the behavior of the system.

So the management wants to validate the actual situation by running the model with the values for the data included in the Technical Specifications Document. The next section includes the results of this initial scenario. Then, the model is run with an increase in the peak percentage. With the analysis of both situations they will feel more comfortable in the negotiation processes with the owners and the labor unions.

\section{INITIAL SCENARIO}

The initial results are included in Table 2. The recorded maximum lengths vary between 9 and 66 users, for 12-meter buses, between 8 and 45, for 15meter buses, and between 2 and 33 for mixed buses, with the exception of line 12, which clearly goes beyond those values.

The main reason is the so-called Congestion Factor. This factor is calculated as the product of the PeakHourInterval (PHI) and the DailyDepartures. It is statistically significant that as the Congestion Factor increases, so does the maximum queue length. The problematic line 12 has a factor of 60 , with the rest not reaching 32.

Out of the two factors that have an impact in the CongestionFactor, the influence of the frequency or PeakHourInterval is counter-intuitive at first. The smaller the frequency (the greater the interval between departures), the longer the queues that are formed. The time in which potential users arrive is longer, since the OpeningGate time is fixed. This is the critical parameter. If the gates are opened only 5 minutes prior to departure and the interval is 30 minutes, 25 minutes out of every 30 are spent in forming queues and 5 to reduce them by jumping into the bus (Figure 4).

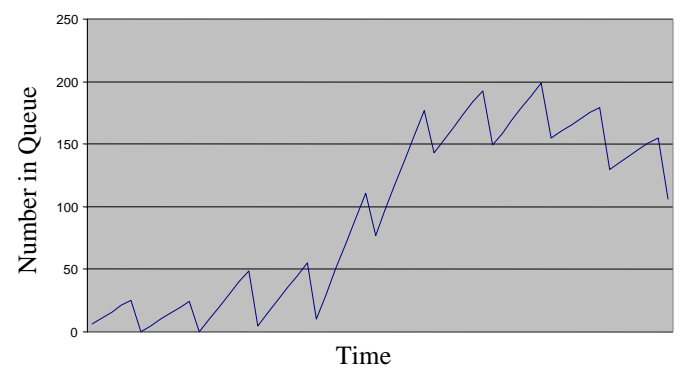

Figure 4. Maximum Queue Length Over Time

Whenever the door is opened, the length of the queue diminishes. When the bus departs, the length of the queue rises again. The problem with this line is that at some point, when the bus departs, there are still people waiting in the queue for the next scheduled bus to arrive.

Table 2. Results for the Current Situation (Peak Percentage $=0.115)$

\begin{tabular}{|c|c|c|c|c|c|c|c|c|}
\hline & \multirow[t]{2}{*}{$\begin{array}{l}\text { Peak Hour } \\
\text { Interval }\end{array}$} & \multirow[t]{2}{*}{ Peak Hour } & \multirow[t]{3}{*}{ Buses } & \multirow[t]{2}{*}{ Daily Departures } & \multirow[t]{2}{*}{ Congestion Factor } & \multicolumn{3}{|c|}{ Maximum Length } \\
\hline & & & & & & & akPercentage 0.1 & \\
\hline & PHI & Departs & & DD & $=\left(\left.\mathrm{PH}\right|^{*} \mathrm{DD}\right) / 1000$ & Bus 12-meters & Bus 15-meters & Bus Mixed \\
\hline 2 & 6 & $8: 00$ & 10 & 2284 & 13.70 & 14 & 9 & 2 \\
\hline 3 & 20 & 7:00 & 3 & 1248 & 24.96 & 41 & 39 & 32 \\
\hline 4 & 20 & $14: 00$ & 3 & 1324 & 26.48 & 43 & 40 & 33 \\
\hline 5 & 15 & $14: 00$ & 4 & 1501 & 22.52 & 35 & 32 & 23 \\
\hline 6 & 30 & $15: 00$ & 2 & 727 & 21.81 & 37 & 36 & 32 \\
\hline 7 & 30 & & 2 & 442 & 13.26 & 23 & 22 & 20 \\
\hline 8 & 30 & & 2 & 561 & 16.83 & 29 & 28 & 25 \\
\hline 10 & 20 & & 3 & 260 & 5.20 & 9 & 8 & 6 \\
\hline 11 & 12 & $14: 00$ & 5 & 2199 & 26.39 & 38 & 34 & 21 \\
\hline 12 & 30 & & 2 & 2111 & 63.34 & 205 & 156 & 94 \\
\hline 13 & 15 & & 4 & 2111 & 31.67 & 66 & 45 & 33 \\
\hline
\end{tabular}


For this reason, the difference in the length of the waiting queues when the capacities of the buses are changed is not significant, but it really is crucial when there is a congestion factor of more than 30. Even the large buses cannot hold the total amount of waiting users.

\section{Sensitivity Analysis for PeakPercentage}

Foreseeing increments in certain days of the year, the system is analyzed for a peak rate of $15 \%$ of the total daily demand. The corresponding results are included in Table 3.

In this case, the necessity of space increases considerably, with line 12 presenting problems, and so do lines 1 and 13 whenever small buses are used.

\section{CONCLUSION}

The static design has been dynamically validated with the use of a simulation model that has been executed to calculate the space required in front of the gates.

Experimentation with the model has allowed for understanding not only the actual system but future situations with higher peaks in demand.

Since the maximum number of waiting passengers are set to 54 (four waiting lines of 14), currently, only line 12 (queue of de 156 users) presents problems if 15meter buses are used for these lines. With mixed buses, the maximum queue length will be 33 passengers, except for line 12 with 94.
For the future, it has been possible to identify several variables that affect the behavior of the system but whose value might only be modified after hard negotiations with labor unions and the owners of the bus lines.

\section{REFERENCES}

Bruzzone A. and G. Signorile. 1999. "Crowd Control Simulation in Java Based Environment", Proceedings of Websim99, San Francisco, USA.

Chalmet, L.G.; Francis, R.L. and P.B. Saunders. 1982. "Network Models for Building Evacuation." Management Science, Vol. 28, No. 1, pp. 86-105

Hanisch, A.; Tolujew, J.; Richter, K. and T. Schulze. 2003. "Online Simulation of Pedestrian Flow in Public Buildings”, Proceedings of the 2003 Winter Simulation Conference, New Orleans, LA.

Hoogendoorn S. P. and M. Hauser. 2004. "Application of Microscopio Pedestrian Flor Simulation to Station Design Evaluation in Lisbon Train Stations", TRB Annual Meeting, Washington, DC, USA.

Li, J. P. 2000. "Train Station Passenger Flow Study", Proceedings of the 2000 Winter Simulation Conference, Orlando, FL.

Otamendi, J. 2005. “A Simulation Model for the Design of the Evacuation Policies in the Case of Fire in Public Integrated Stations”, Proceedings of the 2005 SIS, Brussels.

Teknomo, K. 2006. "Application of Microscopic Pedestrian Simulation Model” Transportation Research Part F 9, $15-27$.

Shen, T.S. 2005. "ESM: a building evacuation simulation model.” Building and Environment 40, No. 5, 671-680.

Table 3. Results for the Future Situation (Peak Percentage $=0.15$ )

\begin{tabular}{|c|c|c|c|c|c|c|c|c|}
\hline & \multirow[t]{2}{*}{$\begin{array}{l}\text { Peak Hour } \\
\text { Interval }\end{array}$} & \multirow[t]{2}{*}{ Peak Hour } & \multirow[t]{3}{*}{ Buses } & \multirow[t]{2}{*}{ Daily Departures } & \multirow[t]{2}{*}{ Congestion Factor } & \multicolumn{3}{|c|}{ Maximum Length } \\
\hline & & & & & & \multicolumn{3}{|c|}{ PeakPercentage 0.115} \\
\hline & PHI & Departs & & DD & $=\left(\left.\mathrm{PH}\right|^{*} \mathrm{DD}\right) / 1000$ & Bus 12-meters & Bus 15-meters & Bus Mixed \\
\hline 1 & 12 & $10: 00$ & 5 & 2609 & 31.31 & 145 & 74 & 33 \\
\hline 2 & 6 & $8: 00$ & 10 & 2284 & 13.70 & 18 & 12 & 2 \\
\hline 3 & 20 & 7:00 & 3 & 1248 & 24.96 & 62 & 50 & 41 \\
\hline 4 & 20 & $14: 00$ & 3 & 1324 & 26.48 & 74 & 53 & 44 \\
\hline 5 & 15 & $14: 00$ & 4 & 1501 & 22.52 & 48 & 42 & 30 \\
\hline 6 & 30 & $15: 00$ & 2 & 727 & 21.81 & 49 & 47 & 42 \\
\hline 7 & 30 & & 2 & 442 & 13.26 & 30 & 29 & 25 \\
\hline 8 & 30 & & 2 & 561 & 16.83 & 89 & 36 & 32 \\
\hline 9 & 12 & $15: 00$ & 5 & 2009 & 24.11 & 63 & 41 & 26 \\
\hline 10 & 20 & & 3 & 260 & 5.20 & 11 & 10 & 8 \\
\hline 11 & 12 & $14: 00$ & 5 & 2199 & 26.39 & 89 & 44 & 28 \\
\hline 12 & 30 & & 2 & 2111 & 63.34 & 347 & 264 & 139 \\
\hline 13 & 15 & & 4 & 2111 & 31.67 & 132 & 80 & 43 \\
\hline
\end{tabular}




\section{AUTHOR BIOGRAPHY}

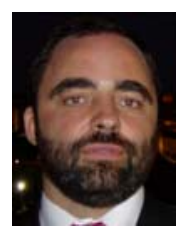

JAVIER OTAMENDI received the B.S.

and M.S. degrees in Industrial Engineering at Oklahoma State University, where he developed his interests in Simulation and Total Quality Management. Back in his home country of Spain, he received a B.S. in Business Administration and a Ph.D. in Industrial Engineering. He is currently a simulation and statistics consultant and university professor at the Rey Juan Carlos University in Madrid. His e-mail address is: jotamendi_30@yahoo.com

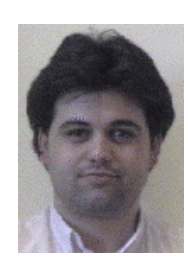

JOSE M. PASTOR received the Electronics and Automatic Control Engineering degree from the Polytechnic University of Madrid (UPM) in 1991 and a Ph.D. in Robotics and Artificial Intelligence in 1997. In 2005 Dr. Pastor moves to the Informatics Systems Department of the Castilla La Mancha University where he is currently Associate Professor. His research interests include intelligent manufacturing systems, and systems simulation and optimization. His e-mail address is: josemanuel.pastor@uclm.es 